\title{
Effects of Separation Processes on Minor Uranium Isotopes in Enrichment Cascades
}

\section{Houston G. Wood}

University of Virginia, Department of Mechanical and Aerospace Engineering, Charlottesville, VA, USA

In natural uranium, there are trace amounts of the "minor isotope" ${ }^{234} \mathrm{U}$ in addition to the more abundant ${ }^{235} \mathrm{U}$ and ${ }^{238} \mathrm{U}$. Uranium irradiated in a reactor and separated from the spent reactor fuel by reprocessing will contain additional minor isotopes. In uranium enrichment, cascades of separating units are tuned to produce the desired level of the isotope ${ }^{235} \mathrm{U}$, but the levels of other isotopes will also change. Measurement of the assays of the ${ }^{235} \mathrm{U}$ in the feed, product and withdrawal streams of each stage allow the calculation of the stage separation factor for the ${ }^{235} \mathrm{U}$. The separation factors for the other isotopes may be calculated. In this aritcle, two methods of modeling the separation factors in a way that represents the physics of two enrichment processes, gas centrifuge and gaseous diffusion, are explored. This technique could be a valuable tool in nuclear forensics. For example, it could be important to know the origin of a sample of highly enriched uranium, found either in intercepted material or in explosive debris. Analysis that could point to the enrichment plant where the uranium was produced could immediately identify weaknesses in safeguards and physical security systems.

\section{INTRODUCTION}

Naturally occurring uranium has three isotopes: ${ }^{234} \mathrm{U},{ }^{235} \mathrm{U}$, and ${ }^{238} \mathrm{U}$. The amounts of each isotope are shown in Table 1.

Uranium enriched in the isotope ${ }^{235} \mathrm{U}$ can be used as fuel for nuclear reactors or, when enriched to higher levels, as a nuclear explosive material in

Received 7 April 2008; accepted 22 June 2008.

The author acknowledges the support of the Program on Science and Global Security at Princeton University where he spent half a year on sabbatical. During this time, the idea of using separation factors based on the physics of the process was suggested by Alexander Glaser and R. Scott Kemp. Their encouragement for this effort is greatly appreciated. The author also thanks Ed Von Halle, who graciously modified his $\mathrm{M}^{*}$ code and provided the code to perform the calculations presented in this article.

Address correspondence to Houston G. Wood, University of Virginia, Department of Mechanical and Aerospace Engineering, 122 Engineer's Way, P. O. Box 400746, Charlottesville, VA 22904-4746, USA. E-mail: hgw9p@virginia.edu 
Table 1: Isotopic concentration of natural uranium. ${ }^{1,2}$

nuclear weapons. There are a number of different methods to enrich uranium. For large-scale enrichment, the two most common methods are gaseous diffusion and gas centrifugation. Currently, there are gaseous diffusion plants in Argentina, China, France, Russia, and the United States. There are gas centrifuge plants known to be in operation or under construction in many countries including Brazil, China, France, Germany, Great Britain, India, Iran, Japan, The Netherlands, Pakistan, Russia, the United States, and the United Kingdom.

In both enrichment processes, a single separating element is insufficient to produce the desired enrichment level or quantity of material. For this reason, separating elements are connected in parallel to form stages (see Figure 1) to produce greater product output. The stages are then connected in series to achieve the greater enrichment levels. These configurations are called cascades (see Figure 2).

Uranium in a nuclear reactor acquires additional minor isotopes inside the reactor. These isotopes include ${ }^{232} \mathrm{U},{ }^{233} \mathrm{U}$, and ${ }^{236} \mathrm{U}$, in addition to the ${ }^{234} \mathrm{U}$ already present. Some of these isotopes have undesirable properties, and if the uranium is to be reused, it is important to understand how their concentrations are increased by further enrichment. Furthermore, these isotopes can play a role in forensic analysis that seeks to determine the source of the uranium or method of enrichment. The concentrations of the minor isotopes in an enriched product stream can be computed by determining their individual unit separation factors, which are simply a measure of how much each isotope is enriched by a single stage.

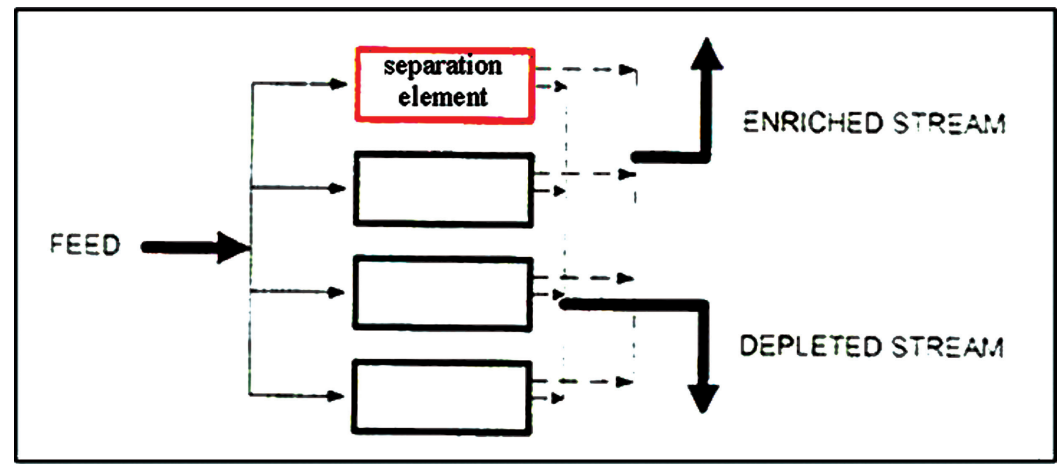

Figure 1: Diagram of an enrichment stage. 


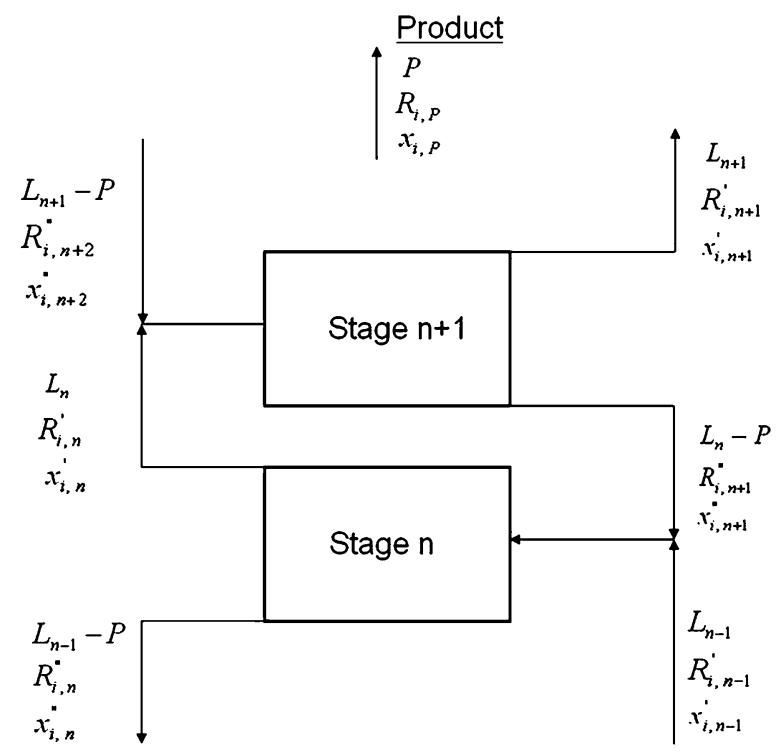

Figure 2: Two adjacent stages in the enriching section of a cascade.

When designing cascades for enriching multicomponent mixtures of uranium, the difference in the assays of the minor isotopes may not be important. However, there are circumstances in which the understanding of these differences may be useful in identifying the separation process by providing a "forensic fingerprint."

This article explores different methods for representing the overall stage separation factors in a multicomponent isotopic mixture. Cascade calculations are performed to study the minor isotopes in LEU and HEU produced from natural and reprocessed uranium gas centrifuge and gaseous diffusion methods.

\section{ANALYSIS AND METHODS FOR CALCULATING MATCHED ABUNDANCE RATIO CASCADES}

The unit separation factor in the gaseous diffusion method is a function of the square root of the ratio of the molecular weights of the component isotopes being separated. The unit separation factor in a gas centrifuge is a function of the difference in the molecular weights. ${ }^{3}$ When analyzing cascades fed with reprocessed uranium for the production of low enriched uranium (LEU) to less than $20 \%{ }^{235} \mathrm{U}$, this distinction is small, but when enriching to HEU (greater than $20 \%{ }^{235} \mathrm{U}$ ), the two processes will produce distinctly different concentrations of the minor isotopes.

The following analysis uses the matched abundance ratio or $\mathbf{M}^{*}$ (read M-star) cascade theory, first suggested by de la Garza. ${ }^{4,5}$ In a cascade that 
separates a binary mixture of isotopes, the assay of the desired isotope in the up-flowing stream from a specific stage is made to match to the assay of the down-flowing stream. This is called a no-mixing cascade or ideal cascade, which also has the desirable feature of minimum inter-stage flow. In a multicomponent mixture, the ideal cascade is generalized to a matched abundance ratio cascade, which will become an ideal cascade when the mixture is binary.

Following Von Halle, ${ }^{6}$ in a multicomponent mixture of $J$ components, let the $k$ th component be designated the "key" component, and let the abundance ratio of each component be defined in terms of the key component by

$$
R_{i}=\frac{x_{i}}{x_{k}} ; i=1,2, \ldots, J
$$

where $R_{i}$ designates the abundance ratio of the $i$ th component and $x_{i}$ denotes the mole fraction of the $i$ th component in the mixture. The overall separation factor for a stage in a cascade is defined as

$$
\alpha_{i}=\frac{R_{i}^{\prime}}{R_{i}^{\prime \prime}} ; i=1,2, \ldots, J
$$

where the superscript (') denotes a quantity in the up-flowing stream leaving a stage and the superscript (") denotes a quantity in the down-flowing stream leaving a stage. This concept is illustrated in Figure 2, which depicts two adjacent stages in the enriching section of a cascade. In the figure, $L_{n}$ is the up-flow from stage number $n$. From the definition, the overall stage separation factor for the key component, $\alpha_{k}$, is unity. In gas centrifuge plants, it is common for cascades to be comprised of centrifuges of the same design and operated at feed flow rates that produce the same separation factor. In this analysis, the overall stage separation factors are assumed to be constant throughout the cascade. That is, all $\alpha_{i}$ are assumed to be independent of the stage number.

The enriching section of the cascade is comprised of all the stages above the feed point, and the stripping section all stages below the feed point. Material balances are taken about both the enriching and stripping sections to describe their performances. The resulting equations are used to describe the performance of the overall cascade.

Computer programs, written in Visual Basic, have been developed by Von Halle $^{7}$ to solve $\mathrm{M}^{*}$ cascade equations. The following input is required: (1) the concentrations of all the isotopes in the feed stream, (2) the concentration of ${ }^{235} \mathrm{U}$ in the both the product and tails stream, (3) either the feed rate or the product rate, and (4) the overall stage separation factor for each isotope. The program then calculates the number of required stages in both the enricher and the stripper and the interstage flow rates.

In the original version of the $\mathrm{M}^{*}$ program, the overall stage separation factor, $\alpha$, for ${ }^{235} \mathrm{U}$ is given as input, and the stage separation factor per unit 
mass difference is calculated as

$$
\alpha_{0}=\alpha^{1 /(238-235)}
$$

Then the overall stage separation factor for the $i$ th component is calculated as

$$
\alpha_{i}=\alpha_{0}^{\left(M_{k}-M_{i}\right)} .
$$

For both gaseous diffusion and gas centrifuge, the process gas is $\mathrm{UF}_{6}$, and the molecular weights of ${ }^{235} \mathrm{UF}_{6}$ and ${ }^{238} \mathrm{UF}_{6}$ are 349 and 352 , respectively. For gaseous diffusion, the overall stage separation factor for ${ }^{235} \mathrm{U}$, not including any inefficiencies, is

$$
\alpha=\sqrt{352 / 349}=1.004289
$$

For a gas centrifuge, the fundamental separation due to the centrifugal force is given by the expression

$$
\exp \left[\Delta M V^{2} / 2 R T\right]=\exp \left[\frac{\Delta M}{M} A^{2}\right],
$$

where $A^{2}=M V^{2} / 2 R T$ is the stratification parameter, $M$ is molecular weight, $T$ is absolute temperature, $\Delta M$ is the difference in molecular weights of the species, and $R$ is the universal gas constant $(8314.4 \mathrm{~J} /(\mathrm{kg} \cdot \mathrm{mole} \cdot \mathrm{K}))$. For the hypothetical "Iguaçu" centrifuge, ${ }^{6,8}$ the peripheral speed is $600 \mathrm{~m} / \mathrm{s}$ and $T=$ $300 \mathrm{~K}$, Eq. (6) yields $A^{2}=25.4$ for $\mathrm{UF}_{6}$. The overall separation factor of a gas centrifuge is determined not only by Eq. (6) but by the feed rate, length of the centrifuge, and other parameters of the countercurrent flow in the centrifuge. Typical centrifuges reported in the literature have overall separation factors on the order of 1.6, considerably larger than gaseous diffusion.

However, if these two values of overall separation factor are used in the classical $\mathrm{M}^{*}$ code, the product and tails concentrations of all the isotopes are the same. The only difference is in the number of stages required in the two cascades. This result is due to the calculation of the overall stage separation factor for the minor isotopes through Eq. (4). Therefore, the $\mathrm{M}^{*}$ code was modified so the user can prescribe all $\alpha_{i}$ in a manner consistent with the separation process being modeled.

\section{DETERMINATION OF SEPARATION FACTORS FOR GAS CENTRIFUGES}

Enrichment of spent reactor fuel by gas centrifuge has been reported ${ }^{9}$ in which the Iquaçu centrifuge parameters were used. In that study, a single gas centrifuge was numerically optimized for enrichment of ${ }^{235} \mathrm{U}$ from a binary mixture of natural uranium.

Then, numerical simulations were performed with spent reactor fuel as feed material using the concentrations of isotopes given in Table 2. The separation 
Table 2: Isotopic concentration of reprocessed uranium. ${ }^{9}$

$\mathrm{U}-232$

$\mathrm{U}-234$

$\mathrm{U}-235$

$\mathrm{U}-236$

U-238
1.E-9 at\%

$0.02 \mathrm{at} \%$

$0.90 \mathrm{at} \%$

0.40 at\%

$98.68 \mathrm{at} \%$

factors were computed as functions of feed rate where the concentration of ${ }^{235} \mathrm{U}$ was maximized with respect to the gradient of the temperature on the rotor wall and drag power of the tails removal scoop. Using the concentrations of the isotopes calculated in the withdrawals streams, the following results were obtained:

$$
\frac{\alpha_{232}-1}{\alpha_{235}-1}=1.97, \quad \frac{\alpha_{234}-1}{\alpha_{235}-1}=1.32, \quad \frac{\alpha_{236}-1}{\alpha_{235}-1}=0.66
$$

These ratios were found to vary by less than $1 \%$ as the feed rate was varied from 1 to $100 \mathrm{mg} / \mathrm{s}$.

Expanding the right-hand side of Eq. (6) in a Taylor series keeping only the first two terms yields

$$
\alpha_{i}-1=\left\{1+\frac{\Delta M_{i}}{M} A^{2}\right\}-1=\frac{\Delta M_{i}}{M} A^{2}
$$

where $\Delta M_{i}$ is the difference in molecular weight between isotope $i$ and the key isotope, in this case ${ }^{238} \mathrm{U}$.

Using Eq. (8) for the isotopes ${ }^{232} \mathrm{U},{ }^{234} \mathrm{U},{ }^{235} \mathrm{U},{ }^{236} \mathrm{U},{ }^{238} \mathrm{U}$, so $J=5$, and taking the key component to be ${ }^{238} \mathrm{U}$, with $k=5$ :

$$
\frac{\alpha_{i}-1}{\alpha_{235}-1}=\frac{M_{i}-M_{k}}{M_{235}-M_{k}}=\frac{\Delta \mathrm{M}_{\mathrm{i}}}{3}, i=1,2, \ldots, 5 .
$$

More terms could be taken in the Taylor series, but this equation predicts values of $2,4 / 3$, and $2 / 3$, which compares very well with the results of the numerical study presented in Eq. (7). If the separation factor for ${ }^{235} \mathrm{U}$ is given, the separation factors for the other components can be determined from the

Table 3: Minor isotope gas centrifuge separation factors from Eqs. (4) and (9) for $\alpha_{235}=1.6$.

\begin{tabular}{lcc}
\hline Isotope & $\boldsymbol{\alpha}_{i}$ from Eq. (9) & $\boldsymbol{\alpha}_{i}$ from Eq. (4) \\
\hline $\mathrm{U}-232$ & 2.2 & 2.5600 \\
$\mathrm{U}-233$ & 2.0 & 2.1888 \\
$\mathrm{U}-234$ & 1.8 & 1.8714 \\
$\mathrm{U}-235$ & 1.6 & 1.6 \\
$\mathrm{U}-236$ & 1.4 & 1.3680 \\
\hline
\end{tabular}


Table 4: Product concentrations in gas centrifuge cascades producing LEU from natural uranium using different $\alpha_{i}$.

\begin{tabular}{lccc} 
Isotope & $\begin{array}{c}\text { Product Concentration } \\
\boldsymbol{\alpha}_{\boldsymbol{i}} \text { from Eq. (9) }\end{array}$ & $\begin{array}{c}\text { Product Concentration } \\
\boldsymbol{\alpha}_{\boldsymbol{i}} \text { from Eq. (4) }\end{array}$ & $\begin{array}{c}\% \\
\text { Difference }\end{array}$ \\
\hline $\mathrm{U}-234$ & 0.00045739 & 0.00047747 & -4.2 \\
$\mathrm{U}-235$ & 0.05 & 0.05 & 0 \\
\hline
\end{tabular}

following:

$$
\alpha_{i}=1+\frac{\Delta M_{i}}{3}\left(\alpha_{235}-1\right), i=1,2, \ldots, 6
$$

The separation factors for gas centrifuges are proportional to mass differences, which is consistent with theoretical results reported elsewhere. ${ }^{11}$ Equation (9) can also be derived by expanding Eq. (4) for the case of overall separation factor near unity.

Table 3 presents the results obtained by Eqs. (4) and (9) to compare results from each method of computing the separation factors for the case where, for convenience, we have chosen $\alpha_{235}=1.6$. The separation factors for isotopes lighter than ${ }^{235} \mathrm{U}$ are greater when calculated by Eq. (4) than by Eq. (9), and the reverse is true for those heavier than ${ }^{235} \mathrm{U}$.

$\mathrm{M}^{*}$ cascade calculations were performed with the modified code for enriching natural uranium to LEU and HEU levels and then for enriching reprocessed uranium to LEU and HEU levels. The feed concentrations for natural and reprocessed uranium used in these calculations are given in Tables 1 and 2 , respectively. In all calculations, the ${ }^{235} \mathrm{U}$ tails concentration was $0.3 \%$.

The results for enriching natural uranium to $5 \%{ }^{235} \mathrm{U}$ are shown in Table 4. The concentration of ${ }^{234} \mathrm{U}$ in the product stream is $4.2 \%$ less when $\alpha_{i}$ are computed by Eq. (9) rather than Eq. (4).

The results for enriching natural uranium to $93 \%{ }^{235} \mathrm{U}$ are shown in Table 5. The difference in ${ }^{234} \mathrm{U}$ concentrations in this case is $4.7 \%$, which is approximately the same percentage difference as in the LEU case (Table 4).

Next, reprocessed uranium as described in Table 2 was used as the feed stream to produce LEU and HEU. The results for the LEU case are presented in Table 6. The differences in the lighter isotope concentrations are 3.8 and $3.3 \%$,

Table 5: Product concentrations in gas centrifuge cascades producing HEU from natural uranium using different $\alpha_{i}$.

\begin{tabular}{lccc} 
Isotope & $\begin{array}{c}\text { Product Concentration } \\
\boldsymbol{\alpha}_{\boldsymbol{i}} \text { from Eq. (9) }\end{array}$ & $\begin{array}{c}\text { Product Concentration } \\
\boldsymbol{\alpha}_{\boldsymbol{i}} \text { from Eq. (4) }\end{array}$ & $\begin{array}{c}\% \\
\text { Difference }\end{array}$ \\
\hline $\mathrm{U}-234$ & 0.00889859 & 0.00933759 & -4.7 \\
$\mathrm{U}-235$ & 0.93 & 0.93 & 0 \\
\hline
\end{tabular}


Table 6: Product concentrations in gas centrifuge cascades producing LEU from reprocessed uranium using different $\alpha_{i}$.

\begin{tabular}{lccc} 
Isotope & $\begin{array}{c}\text { Product Concentration } \\
\boldsymbol{\alpha}_{\boldsymbol{i}} \text { from Eq. (9) }\end{array}$ & $\begin{array}{c}\text { Product Concentration } \\
\boldsymbol{\alpha}_{\boldsymbol{i}} \text { from Eq. (4) }\end{array}$ & $\begin{array}{c}\% \\
\text { Difference }\end{array}$ \\
\hline$U-232$ & $7.2635 \mathrm{E}-11$ & $7.5514 \mathrm{E}-11$ & -3.8 \\
$\mathrm{U}-234$ & 0.00128628 & 0.00132990 & -3.3 \\
$\mathrm{U}-235$ & 0.05 & 0.05 & 0 \\
$\mathrm{U}-236$ & 0.01673063 & 0.01567094 & +6.8 \\
\hline
\end{tabular}

which is approximately the same percentage difference as with the natural uranium feed. However, the ${ }^{236} \mathrm{U}$ concentration is considerably greater at $+6.8 \%$.

For enriching the reprocessed uranium in Table 2 to HEU levels, the assay of $93 \%{ }^{235} \mathrm{U}$ could not be obtained using either set of separation factors. This is due to the high concentration of ${ }^{236} \mathrm{U}$ in the sample material. In this case, enrichment to $75 \%{ }^{235} \mathrm{U}$ was about as high as could be achieved (see Table 7). The percentage difference in the lighter isotopes is 3.9 and 3.8\% less using Eq. (9), almost the same as in the LEU case (see Table 6). However, the percentage difference in ${ }^{236} \mathrm{U}$ concentration was $+17.9 \%$, almost three times the percentage difference in the LEU case.

\section{DETERMINATION OF SEPARATION FACTORS FOR GASEOUS DIFFUSION}

For gaseous diffusion, we use Eq. (5) to determine the separation factors for all the isotopes

$$
\alpha_{i}=\sqrt{M_{k} / M_{i}}, i=1, \ldots, J
$$

where $M_{i}$ is the molecular weight of each isotope and $M_{k}$ is the molecular weight of the key component. For ${ }^{238} \mathrm{UF}_{6}, M_{k}=352$.

The $\alpha_{i}$ are calculated from Eq. (4), as in the original $\mathrm{M}^{*}$ code, and by Eq. (10) and presented in Table 8. For the small $\alpha$ of gaseous diffusion, the values obtained by the two codes are much closer than in the large $\alpha$ of gas centrifuge shown in Table 3.

Table 7: Product concentrations in gas centrifuge cascades producing HEU from reprocessed uranium using different $\alpha_{i}$.

\begin{tabular}{lccc} 
Isotope & $\begin{array}{c}\text { Product Concentration } \\
\boldsymbol{\alpha}_{\boldsymbol{i}} \text { from Eq. (9) }\end{array}$ & $\begin{array}{c}\text { Product Concentration } \\
\boldsymbol{\alpha}_{\boldsymbol{i}} \text { from Eq. (4) }\end{array}$ & $\begin{array}{c}\% \\
\text { Difference }\end{array}$ \\
\hline $\mathrm{U}-232$ & $1.1531 \mathrm{E}-09$ & $1.1999 \mathrm{E}-09$ & -3.9 \\
$\mathrm{U}-234$ & 0.02016983 & 0.02095960 & -3.8 \\
$\mathrm{U}-235$ & 0.75 & 0.75 & 0 \\
$\mathrm{U}-236$ & 0.19342117 & 0.16406253 & +17.9 \\
\hline
\end{tabular}




\section{Wood}

Table 8: Minor isotope gaseous diffusion separation factors from Eqs. (4) and (10) for $\alpha_{235}=1.004289$.

\begin{tabular}{lcc}
\hline Isotope & $\alpha_{i}$ from Eq. (10) & $\alpha_{i}$ from Eq. (4) \\
\hline U-232 & 1.008633 & 1.008596 \\
U-233 & 1.007179 & 1.007159 \\
U-234 & 1.005731 & 1.005723 \\
U-235 & 1.004289 & 1.004289 \\
U-236 & 1.002853 & 1.002857 \\
\hline
\end{tabular}

Table 9: Product concentrations in gaseous diffusion cascades producing LEU from natural uranium using different $\alpha_{i}$.

\begin{tabular}{lccc} 
Isotope & $\begin{array}{c}\text { Product Concentration } \\
\boldsymbol{\alpha}_{\boldsymbol{i}} \text { from Eq. (10) }\end{array}$ & $\begin{array}{c}\text { Product Concentration } \\
\boldsymbol{\alpha}_{\boldsymbol{i}} \text { from Eq. (4) }\end{array}$ & $\begin{array}{c}\% \\
\text { Difference }\end{array}$ \\
\hline $\mathrm{U}-234$ & 0.00047791 & 0.00047747 & -.0922 \\
$\mathrm{U}-235$ & 0.05 & 0.05 & 0 \\
\hline
\end{tabular}

Table 10: Product concentrations in gaseous diffusion cascades producing HEU from natural uranium using different $\alpha_{i}$.

\begin{tabular}{lccc} 
Isotope & $\begin{array}{c}\text { Product Concentration } \\
\boldsymbol{\alpha}_{\boldsymbol{i}} \text { from Eq. (10) }\end{array}$ & $\begin{array}{c}\text { Product Concentration } \\
\boldsymbol{\alpha}_{\boldsymbol{i}} \text { from Eq. (4) }\end{array}$ & $\begin{array}{c}\% \\
\text { Difference }\end{array}$ \\
\hline $\mathrm{U}-234$ & 0.00934694 & 0.00933759 & -0.100 \\
$\mathrm{U}-235$ & 0.93 & 0.93 & 0 \\
\hline
\end{tabular}

Table 11: Product concentrations in gaseous diffusion cascades producing LEU from reprocessed uranium using different $\alpha_{i}$.

\begin{tabular}{lccc} 
Isotope & $\begin{array}{c}\text { Product Concentration } \\
\boldsymbol{\alpha}_{\boldsymbol{i}} \text { from Eq. (10) }\end{array}$ & $\begin{array}{c}\text { Product Concentration } \\
\boldsymbol{\alpha}_{\boldsymbol{i}} \text { from Eq. (4) }\end{array}$ & $\begin{array}{c}\% \\
\text { Difference }\end{array}$ \\
\hline $\mathrm{U}-232$ & $7.5567 \mathrm{E}-11$ & $7.5514 \mathrm{E}-11$ & -0.070 \\
$\mathrm{U}-234$ & 0.00133083 & 0.00132990 & -.070 \\
$\mathrm{U}-235$ & 0.05 & 0.05 & 0 \\
$\mathrm{U}-236$ & 0.01564999 & 0.01567094 & 0.134 \\
\hline
\end{tabular}

Table 12: Product concentrations in gaseous diffusion cascades producing HEU from reprocessed uranium using different $\alpha_{i}$.

\begin{tabular}{lccc} 
Isotope & $\begin{array}{c}\text { Product Concentration } \\
\boldsymbol{\alpha}_{\boldsymbol{i}} \text { from Eq. (10) }\end{array}$ & $\begin{array}{c}\text { Product Concentration } \\
\boldsymbol{\alpha}_{\boldsymbol{i}} \text { from Eq. (4) }\end{array}$ & $\begin{array}{c}\% \\
\text { Difference }\end{array}$ \\
\hline $\mathrm{U}-232$ & $1.2008 \mathrm{E}-09$ & $1.1999 \mathrm{E}-09$ & -0.075 \\
$\mathrm{U}-234$ & 0.02097610 & 0.02095960 & -0.079 \\
$\mathrm{U}-235$ & 0.75 & 0.75 & 0 \\
$\mathrm{U}-236$ & 0.16348610 & 0.16406253 & 0.351 \\
\hline
\end{tabular}


With the two sets of $\alpha$ shown in Table 8, the same series of calculations performed for gas centrifuges is repeated for gaseous diffusion. Table 9 presents the product concentrations with the two sets of separation factors representing gaseous diffusion enriching natural uranium to LEU. The percentage difference for ${ }^{234} \mathrm{U}$ in the product stream is less than $0.1 \%$. Table 10 presents the results of producing HEU from natural uranium by gaseous diffusion, and the difference in ${ }^{234} \mathrm{U}$ in the product stream is exactly $0.1 \%$.

Results of enriching reprocessed uranium to LEU and HEU are presented in Tables 11 and 12, respectively. In both of these cases, the difference in computed assays for the isotopes lighter than ${ }^{235} \mathrm{U}$ is less than $0.1 \%$. The ${ }^{236} \mathrm{U}$ assays differ by $0.134 \%$ in LEU and by $0.351 \%$ in HEU. These results are strikingly different than those presented for the gas centrifuge cascades.

\section{CONCLUSIONS AND RECOMMENDATIONS}

In this article, we have attempted to model the separation factors in a way that represents the physics of two enrichment processes, gas centrifuge and gaseous diffusion. Different methods for representing the overall stage separation factors, $\alpha_{i}$, for the ith component in a multicomponent isotopic mixture have been analyzed, and cascade calculations have been performed using a modified $\mathbf{M}^{*}$ code to study the minor isotopes in LEU and HEU produced from natural uranium and reprocessed uranium.

In a gas centrifuge, the separation factors depend on the difference in molecular weights and are modeled by Eq. (9). In gaseous diffusion, the separation factors depend on the ratio of molecular weights and are modeled by Eq. (10). The original $\mathrm{M}^{*}$ code was modified to allow the user to prescribe the separation factors for each of the isotopes.

The results of the calculations presented above show there is a significant, and perhaps measurable, difference in predicted concentrations of the minor isotopes in both LEU and HEU material produced by gas centrifuge and gaseous diffusion. The results suggest the minor isotopes have potential to be a forensic marker in enriched uranium.

Because the minor isotopes of uranium have the potential to be a useful forensic tool, it is desirable to validate the theoretical models presented in this article. Analysis that could point to the enrichment plant where the uranium was produced could immediately identify weaknesses in safeguards and physical security systems. To this end, it would be very valuable to have experimental data for reprocessed uranium enriched by gas centrifuges to validate the mathematical model described by Eq. (9) and the $\mathrm{M}^{*}$ cascade calculations. 


\section{NOTES AND REFERENCES}

1. S. Richter, A. Alonso, W. De bolle, R. Wellum, and P. D. P. Taylor, "Isotopic 'Fingerprints' for Natural Uranium Ore Samples," International Journal of Mass Spectrometry, 193(1999), 9-14.

2. U.S. Department of Energy Nuclear Material Safeguards for Uranium Enrichment Plats. Technical Publication No. ISPO-347/R6. (Oak Ridge, TN, Oak Ridge National Laboratory, 2005).

3. J. Shacter, R. L. Hoglund, and E. Von Halle, "Diffusion Separation Methods," Encyclopedia of Chemical Technology (John Wiley \& Sons, Inc., 1965), 91-175.

4. A. De La Garza, G. A. Garrett, and J. E. Murphy, "Multicomponent Isotope Separation in Cascades," Chemical Engineering Science, 15(1961), 188-209.

5. A. De La Garza, "A Generalization of the Matched Abundance-Ratio Cascade for Multicomponent Isotope Separation," Chemical Engineering Science 18(1963), 73-82.

6. E. Von Halle "Multicomponent Isotope Separation in Matched Abundance Ratio Cascades Composed of Stages with Large Separation Factors" in Proceedings of the 1st Workshop on Separation Phenomena in Liquids and Gases, ed. K. G. Roesner (Darmstadt, Germany, Institut für Mechanik, 1987), 325-356.

7. E. Von Halle, personal communication (2007), December 1-10, 2007.

8. C. Schwab, N. A. S. Rodrigues, and H. G. Wood, eds., Fifth Workshop Proceedings Separation Phenomena in Liquids and Gases, Centro Técnico Aeroespacial, Instituto de Estudos Avançados, Ministério da Ciência e Tecnologia, Instituto Nacional de Pesquisas Espaciai (Foz do Iguaçu, Brasil, September 22-26, 1996).

9. F. Doneddu, P. Roblin, and H. G. Wood, "Optimization Studies for Gas Centrifuges," Separation Science and Technology, 35(2000), 1207-1221.

10. Frederic Doneddu, Philippe Roblin, and Houston G. Wood, "Optimization Studies for Gas Centrifuges," Separation Science and Technology, 35 (2000), 8, 1207-1221.

11. R. S. Kemp, "Gas Centrifuge Theory and Development: A Review of the U.S. Approach," Science and Global Security, publication pending. 